\title{
Stabilization of wide band-gap p-type wurtzite MnTe thin films on amorphous substrates
}

Sebastian Siol ${ }^{1,2 *}$, Yanbing Han ${ }^{1,3}$, John Mangum ${ }^{4}$, Philip Schulz ${ }^{1+}$, Aaron M. Holder ${ }^{1}$, Talysa R. Klein ${ }^{1}$, Maikel F. A. M. van Hest ${ }^{1}$, Brian Gorman ${ }^{4}$, Andriy Zakutayev $^{1 *}$

${ }^{1}$ National Renewable Energy Laboratory, Golden, CO 80401, USA

${ }^{2}$ EMPA - Swiss Federal Laboratories for Materials Science and Technology,

\author{
8600 Dübendorf, Switzerland \\ ${ }^{3}$ Fudan University, Shanghai 200433, China \\ ${ }^{4}$ Colorado School of Mines, Golden, CO 80401, USA
}

*Corresponding authors: Andriy.Zakutayev@nrel.gov, Sebastian.Siol@empa.ch

${ }^{+}$Current address: CNRS-Institut Photovoltaique d'Ile de France (IPVF), UMR 9006, Palaiseau, France

Keywords: WZ-MnTe, polymorphism, chemical stabilization, photoelectron spectroscopy, XPS, UPS, TEM, wurtzite, nickeline, sputtering

\begin{abstract}
An important challenge in the development of optoelectronic devices for energy conversion applications is the search for suitable p-type contact materials. For example, p-type MnTe would be a promising alternative back contact to due to their chemical compatibility, but at normal conditions it has too narrow band gap due to octahedrally coordinated nickeline (NC) structure. The tetrahedrally coordinated wurtzite (WZ) polymorph of MnTe has not been reported, but it is especially interesting due to its predicted wider band gap, and because of better structural compatibility with CdTe and related II-VI semiconductor materials. Here, we report on the stabilization of WZ-MnTe thin films on amorphous indium zinc oxide (a-IZO) substrates relevant to photovoltaic applications. Optical spectroscopy of the WZ-MnTe films shows a wide direct band gap of $E_{\mathrm{g}}=2.7 \mathrm{eV}$, while PES measurements reveal weak p-type doping with the Fermi level $0.6 \mathrm{eV}$ above the valence band maximum. The results of electron microscopy and photoelectron spectroscopy (PES) measurements indicate that the WZ-MnTe is stabilized due to interdiffusion at the interface with IZO. The results of this work introduce a substrate stabilized WZ-MnTe polymorph as a potential p-type contact material candidate for future applications in CdTe devices for solar energy conversion and other optoelectronic technologies.
\end{abstract}

This document is the accepted manuscript version of the following article: Siol, S., Han, Y., Mangum, J., Schulz, P., Holder, A., Klein, T. R., ... Zakutayev, A. (2018). Stabilization of wide band-gap p-type wurtzite MnTe thin films on amorphous substrates. Journal of Materials Chemistry C. http://doi.org/10.1039/C8TC01828F 


\section{Introduction}

Main group II-VI compound semiconductors such as $\mathrm{CdTe}, \mathrm{ZnO}, \mathrm{CdS}, \mathrm{ZnTe}$, are important materials for optoelectronic applications such as solar energy conversion. All these materials crystalize in tetrahedrally bonded zincblende (ZB) or wurtzite (WZ) structures ${ }^{1,2}$. A key challenge in developing and improving semiconductor devices based on these materials is the search for suitable contacts. Important criteria when choosing a contact material are suitable optoelectronic properties, chemical compatibility, and structural mismatch at the interface. However, many potentially interesting contact materials that are chemically compatible with IIVI semiconductors crystallize in octahedrally coordinated structures, such as rocksalt (RS) for $\mathrm{MnS}$ or $\mathrm{MnSe}^{1}$. Stabilizing their tetrahedrally coordinated polymorphs would be useful due to their better structural compatibility with the II-VI semiconductors. An interesting test case material in this context for which such polymorphs have been reported is manganese telluride $(\mathrm{MnTe})^{3-5}$. MnTe has been extensively studied in the past for its application in dilute magnetic semiconductors (DMS) $)^{6,7}$. In recent years MnTe has been mentioned as a promising back contact material for CdTe thin film photovoltaic solar cells ${ }^{8}$, where development of alternative $\mathrm{Cu}$-free back contacts is needed ${ }^{9}$.

Manganese telluride MnTe is distinct from other manganese chalcogenides $\mathrm{MnCh}(\mathrm{Ch}=\mathrm{O}, \mathrm{S}, \mathrm{Se})$ due to its different ground state crystal structure. At normal temperature and pressure MnTe crystallizes in the octahedrally coordinated NiAs-type nickeline structure ${ }^{1}(\mathrm{NC})$, while $\mathrm{MnTe}_{2}$ crystallizes in the pyrite/FeS ${ }_{2}$ structure $(\mathrm{PY})^{10}$. Other polymorphs of MnTe have been predicted ${ }^{11}$ (see Figure 1) but can only be stabilized under high pressure or using non-equilibrium synthesis methods. For example $\mathrm{MnTe}$ in $\mathrm{NaCl}(\mathrm{RS})$ and wurtzite (WZ) structure could be stabilized under high-pressure conditions in a diamond anvil ${ }^{3,4}$, while thin films of ZB-type MnTe could be synthesized using molecular beam epitaxy (MBE) methods ${ }^{5}$. The tetrahedrally coordinated non-equilibrium polymorphs zincblende (ZB) and wurtzite (WZ) are rare, but especially interesting due to their wider band gaps ${ }^{12}$ and their structural compatibility with CdTe and other II-VI semiconductors. However the WZ-type MnTe - even though it has been calculated to have low energy above the ground state and synthesized at high pressures - has not been experimentally stabilized at normal conditions, relevant to optoelectronic applications. 


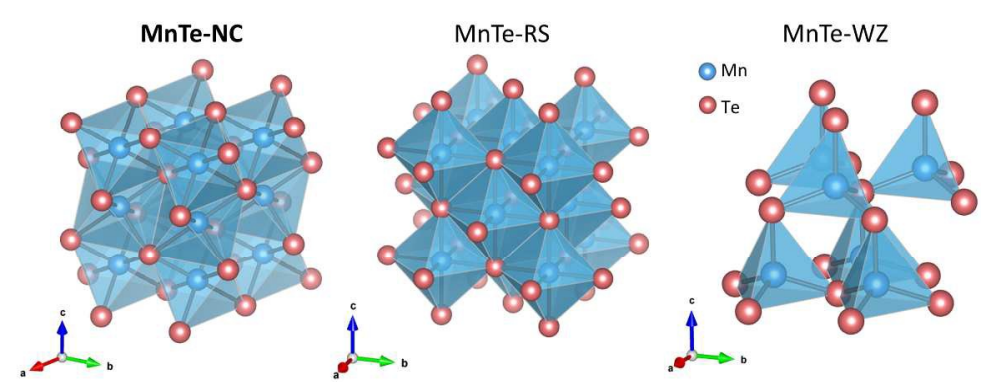

Figure 1: Crystal structures of different MnTe polymorphs: At normal conditions MnTe crystallizes in the octahedrally bonded nickeline (NC) structure (bold font). Other possible polymorphs include the octahedrally bonded rock salt (RS)- and tetrahedrally bonded wurtzite (WZ) structures.

In this work we report on the stabilization of MnTe films in WZ structure on amorphous IZO substrates (a-IZO) using non-equilibrium sputter deposition. Due to the amorphous structure of the underlying film, substrate epitaxial templating effects can be ruled out. In addition, the WZMnTe film thicknesses of over $600 \mathrm{~nm}$ exceed the critical values typically observed for films obtained through strain induced stabilization. Instead, the stabilization of the WZ polymorph is the result of inter-diffusion processes, as indicated by detailed studies of the chemical and structural properties of the interfacial region. Measurements of the optoelectronic properties of the resulting WZ-type MnTe polymorph show weak p-type doping and an increased direct band gap of $2.7 \mathrm{eV}$ compared to the NC-type MnTe ground state structure. The results of this work introduce a new functional polymorph of this interesting semiconducting compound with potential applications as a wide band gap contact layer in optoelectronic devices.

\section{Results}

\subsection{Structural and optical characterization}

Sputtered MnTe films on glass substrates typically crystallize in the NC ground state structure 13,14. Curiously, upon sputtering on n-type amorphous indium zinc oxide (a-IZO) layers a stabilization of the WZ polymorph was observed. Figure 2a shows wide-angle X-ray diffraction (XRD) measurements of up to $600 \mathrm{~nm}$ thick MnTe films on various substrates. The measured diffraction pattern are in good agreement with calculated reference patterns ${ }^{12}$ for $\mathrm{NC}$ - and WZ- 
type MnTe, respectively. Slight variations in the measured peak position compared the calculated reference patterns can be caused by a small, but systematic, uncertainty in theoretical calculation $^{15}$ and residual stress in the thin films. Note that these shifts are present in both WZMnTe/a-IZO and NC-MnTe/SiO ${ }_{2}$ samples. The missing reflections in the XRD patterns of the WZ-type films can be explained by a preferential orientation of the WZ-type films. Detector images of the films on a-IZO show preferential orientation toward the growth axis (see Figure 2b), an effect commonly observed for sputter deposited thin films ${ }^{16,17}$. Compositional analysis using X-ray fluorescence measurements shows anion/cation-ratios close to unity confirming the stoichiometry of the MnTe films. No XRD peaks related to $\mathrm{ZnO}$ or $\operatorname{In}_{2} \mathrm{O}_{3}$ that can be crystallization products of a-IZO are observed.

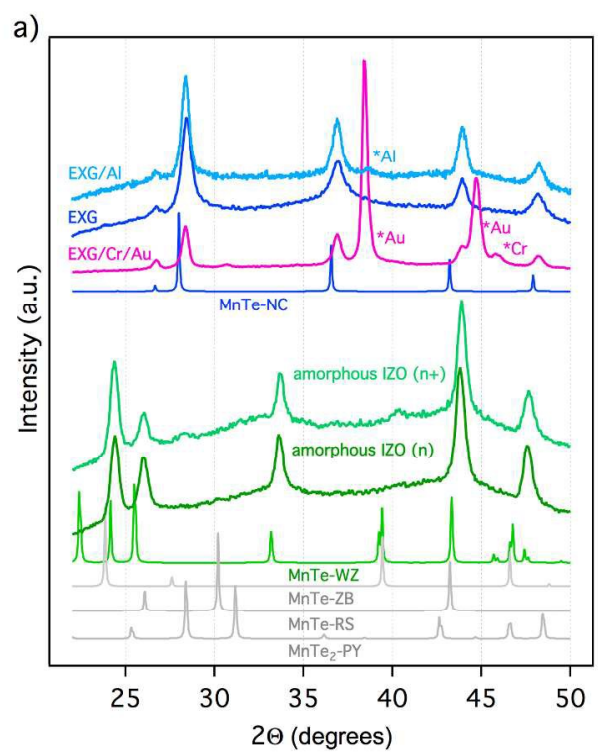

b)

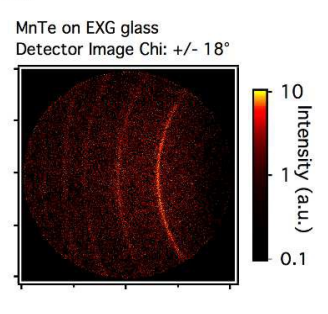

MnTe on amorphous IzO Detector Image Chi: $+/-18^{\circ}$

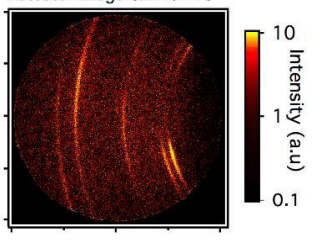

Figure 2: a) Wide-angle X-ray diffraction measurements of MnTe films deposited on different substrates at $320^{\circ} \mathrm{C}$. On amorphous IZO coated glass substrates the films crystallize in WZ structure. b) 2D detector images indicate preferential orientation of the WZ-MnTe films.

Considering the amorphous structure of the underlying a-IZO and film thicknesses in excess of $600 \mathrm{~nm}$, the epitaxial templating and strain stabilization effects can be ruled out. As an alternative hypothesis, it was investigated if the stabilization mechanism is rather induced by charge transfer from the substrate. To test this hypothesis MnTe thin films were sputtered on metals with different work functions, specifically $\mathrm{Au}(5.1 \mathrm{eV})$ and $\mathrm{Al}(4.1 \mathrm{eV})$. Despite the large difference in the substrate's surface potential no difference in the measured NC crystal structure 
could be observed. On plain Eagle XG borosilicate glass (EXG), the MnTe films also crystallized in NC structure. Only on a-IZO, regardless of the doping level, the films crystallize in WZ-structure. Thus mechanisms other than epitaxial templating, strain stabilization, or charge transfer must be leading to formation of WZ-MnTe on a-IZO substrates.

One of the most apparent differences between the NC- and WZ-type MnTe films is the optical bandgap (Figure 3). Figure 3d and Figure 3e show photographs of MnTe films grown on glass as well as on a-IZO substrates. A strong contrast in optical absorption occurs between areas that were coated with a-IZO prior to the MnTe deposition and those without a-IZO coating. UV-Vis spectroscopy confirms the optical impression. A significant change in absorption onset occurs upon crystallization in the wurtzite structure (Figure 3a). Figure $3 \mathrm{~b}$ and $3 \mathrm{c}$ show Tauc plots for the determination of the direct and indirect band gaps, respectively. For NC-type MnTe the indirect band gap of $1.25 \mathrm{eV}$ is in good agreement with the reported literature value of $\sim 1.27$ $\mathrm{eV}^{18}$. For WZ-type MnTe an indirect band gap of $2.4 \mathrm{eV}$ as well as a direct band gap $2.7 \mathrm{eV}$ could be determined, being consistent with band gap values from GW calculations ${ }^{14}$. Some absorption below these photon energies are observed, likely due to combination in measurement limitation (lack of diffuse scattering collection) and sub-gap defect states in these samples. JVmeasurements on a-IZO/MnTe/Au device stacks show weak rectifying behavior consistent with the weak p-type doping of the WZ-MnTe films (see Figure S1 in supporting information). The resistivity could be estimated to be on the order of a few $1000 \Omega \mathrm{cm}$, which is in good agreement with the increase in band gap. For comparison NC-MnTe measures around $1 \Omega \mathrm{cm}^{14}$; such large difference is unlikely to result from the elemental diffusion alone.
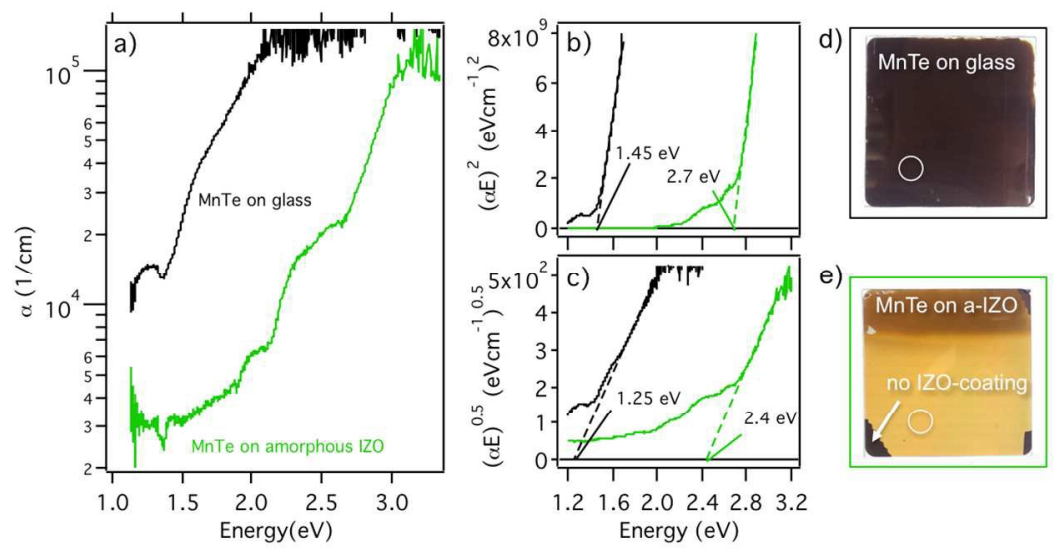
Figure 3: UV-Vis spectroscopy results for MnTe films grown on glass and a-IZO at $320^{\circ} \mathrm{C}$ : a) absorption coefficient $\alpha$; b) Tauc-plot $(\alpha E)^{2}$ for the determination of the direct band gap; c) Taucplot $(\alpha \mathrm{E})^{1 / 2}$ for the determination of the indirect band gap; d), e) Photographs of 50 x $50 \mathrm{~mm}$ MnTe films (500nm thickness) with backlight illumination. The measurement spots are indicated with circles.

\subsection{Electron microscopy}

To investigate the stabilization mechanism of WZ-MnTe, as well as the nanostructure of the MnTe films, detailed scanning / transmission electron microscopy ((S)TEM) was performed on MnTe films grown on glass as well as a-IZO (Figure 4). Figure 4a and Figure 4e show selected area electron diffraction (SAED) patterns of MnTe grown on glass and a-IZO, respectively, indicating that both MnTe films are polycrystalline, whereas the IZO layer is amorphous (consistent with XRD results). On glass MnTe crystallizes in NC-structure, on a-IZO the majority phase is WZ-type MnTe. Besides the WZ-type MnTe, diffraction peaks for PY-MnTe 2 and potentially ZB-MnTe are visible on a-IZO substrates. These secondary phases are likely formed due to inter-diffusion processes at the a-IZO/MnTe interface, as shown by $10 \mathrm{~nm}$ thick region in Figure 4f. In contrast, high-resolution TEM imaging of the interfacial regions on glass show a well-defined interface for MnTe grown (Figure 4b) Additional images can be found in supporting information (Figure S2).

Energy-dispersive X-ray spectroscopy (EDS) of the interfacial regions confirms the presence of diffusion processes at the interface. EDS maps of MnTe grown on a-IZO show a Mn-rich interfacial layer followed by a Te-rich depletion region, which is consistent with the presence of $\mathrm{MnTe}_{2}$ in the film (Figure 4e). Quantitative line scans indicate Mn-diffusion into the a-IZO layer as well as significant amounts of In- and Zn-diffusion into the MnTe layer. Diffusion of Mn and $\mathrm{Zn}$ appears to be localized within a range of $10 \mathrm{~nm}-20 \mathrm{~nm}$ around the interface, while In seems to diffuse even further into the MnTe-film. Due to the nature of the microscopy measurement technique an increased surface roughness of the substrate may result in integration over several offset atoms, which may lead to an apparent broadening of the interfacial region. However, this effect can be ruled out since AFM studies on similarly processed a-IZO layers showed a surface 
roughness well below $1 \mathrm{~nm}^{19}$. For the films on glass no such inter-diffusion can be observed, instead an overlap in the $\mathrm{Si}$ as well as $\mathrm{Mn}$ and Te emissions results in overestimated Siconcentrations in the MnTe layer.
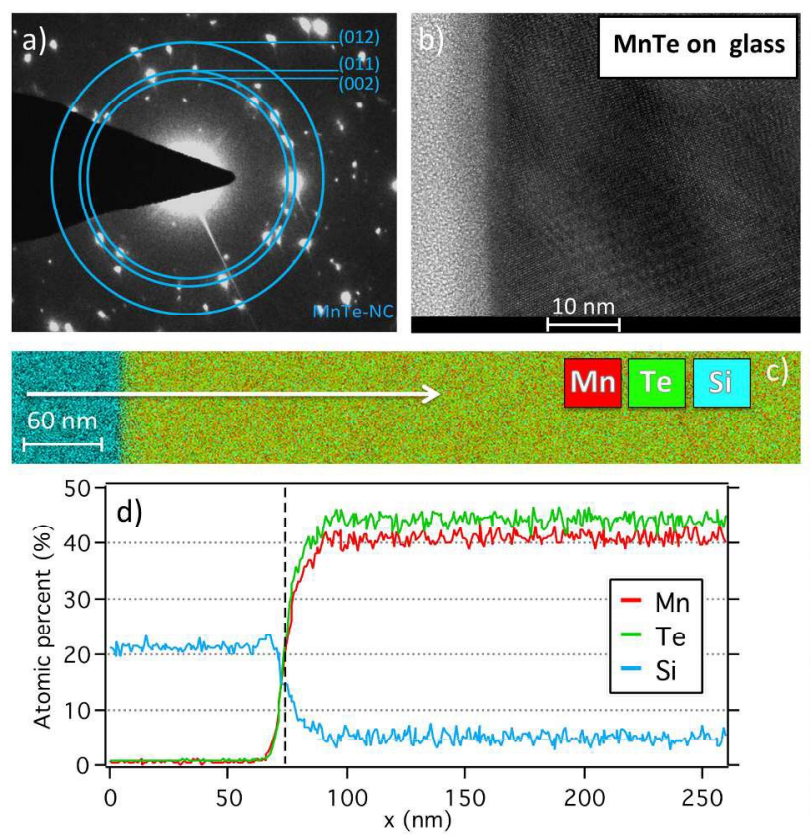
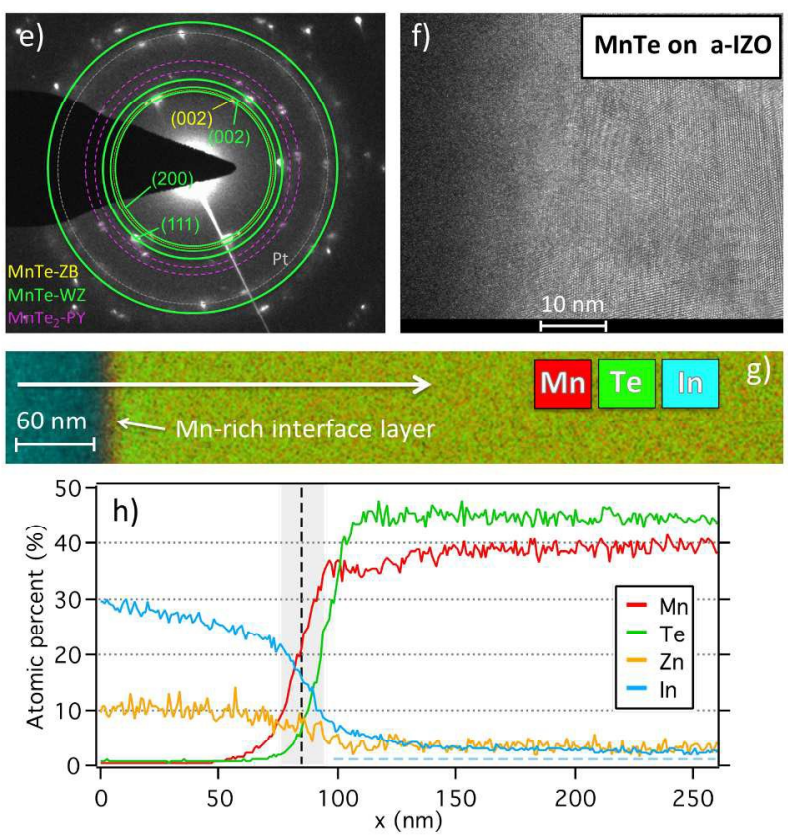

Figure 4: (S)TEM analysis of MnTe grown on glass and on a-IZO: a),e) SAED of MnTe on glass shows mainly NC-type structure, on a-IZO several structures can be observed. Besides WZ-type MnTe, diffraction peaks for PY-MnTe 2 and potentially ZB-MnTe are visible. b),f) High resolution TEM micrographs of the interfacial region. c),g) STEM-EDS analysis of the interface regions. At the a-IZO/MnTe interface a Mn-rich interfacial layer can be observed. d),h) Quantitative results of EDS line scans over both interfaces indicating inter-diffusion at the aIZO/MnTe interface.

\subsection{Photoelectron spectroscopy interface analysis}

To further elucidate the chemical processes that led to inter-diffusion at the a-IZO/MnTe interface observed by EDS, a detailed photoelectron spectroscopy (PES) study was carried out. An inter-tool vacuum transport from the deposition chamber to the measurement system was 
used to perform the interface study at controlled atmosphere and minimal surface contamination (see method section). X-ray photoelectron spectroscopy (XPS) spectra were measured for core level emissions of In $3 \mathrm{~d}_{5 / 2}, \mathrm{Zn} 2 \mathrm{p}_{3 / 2}, \mathrm{O}_{1 \mathrm{~s}}$ as well as Mn $2 \mathrm{p}_{3 / 2}$, Te $3 \mathrm{~d}_{5 / 2}$ and the valence band (VB).

Figure 5 shows detailed spectra of these core level emissions for varying MnTe film thickness.Figure 5 shows detailed spectra of these core level emissions for varying MnTe film thickness. The areal intensity of the core level emissions weighted by their respective atomic sensitivity factors gives valuable insight into the chemical composition of the films surface. From the relative positions and the shifts of these peaks, chemical reactions and band offsets at the interface can be deduced.

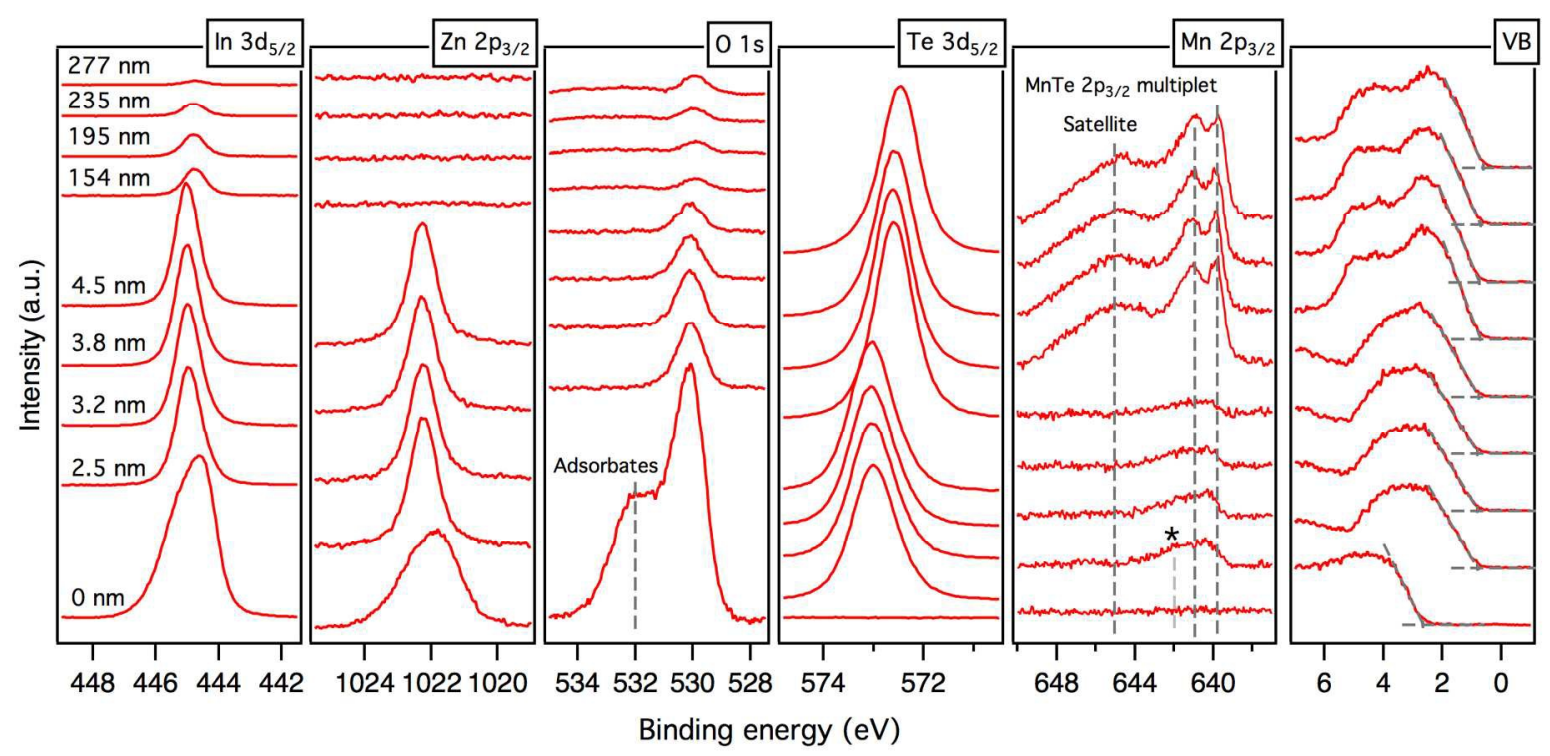

Figure 5: Detailed XP spectra of all relevant core level emissions as well as the valence band region for different MnTe film thicknesses. As the MnTe thickness increases an attenuation of the $\mathrm{In}, \mathrm{Zn}$ and $\mathrm{O}$ related emissions is observed. A strong shift in the $\mathrm{Te} 3 \mathrm{~d}_{5 / 2}$ core level binding energy is observed from the first to the second MnTe deposition cycle. *The broadening in the $\mathrm{Mn} 2 \mathrm{p}_{3 / 2}$ core level emission could indicate presence of $\mathrm{Mn}^{4+}$.

Figure 6a shows the composition of the film surface as a function of MnTe film thickness. The strong $\mathrm{Zn}$ and $\mathrm{In}$ signals for low film thicknesses up to $4.5 \mathrm{~nm}$ indicate $\mathrm{In}$ and $\mathrm{Zn}$ diffusion into the MnTe film. This observation could be also due to incomplete coverage of the substrate, but 
given the low roughness of the a-IZO layer ${ }^{19}$ this is unlikely. While the $\mathrm{Zn}$ diffusion appears to occur on a scale of a few $\mathrm{nm}$, In can be measured further into the film. More striking is the change of the $\mathrm{Mn} / \mathrm{Te}$ ratio with increasing MnTe film thickness. Close to the interface the films are Te-rich, which is in good agreement with an analysis of the Mn 2 $\mathrm{p}_{3 / 2}$ core level emission. In MnTe the charge state is $\mathrm{Mn}^{2+}$, which results in a strong satellite emission at around $645 \mathrm{eV}$ binding energy ${ }^{5,20}$ as can be seen for film thicknesses $>154 \mathrm{~nm}$. This satellite emission is comparatively weak for film thicknesses below $4.5 \mathrm{~nm}$ indicating the presence and potential diffusion of metallic $\mathrm{Mn}$ at the interface. In addition a broadening of the $\mathrm{Mn}_{2 \mathrm{p} 3 / 2}$ core level emission could indicate the presence of $\mathrm{Mn}^{4+}\left(\sim 642 \mathrm{eV}^{20}\right)$, being consistent with the presence of $\mathrm{MnTe}_{2}$ at the interface (see SAED-analysis, Figure 4e).

Figure 6b shows the evolution of the individual core level binding energies (CLBE) with increasing MnTe film thickness. For well-defined interfaces without interfacial reactions CLBE of elements associated with the either substrate or film typically shift in parallel. In the present case the Te $3 \mathrm{~d}$ CLBE as well as the valence band maximum of MnTe in particular show a different evolution with increasing film thickness, supporting the presence of secondary phases such as $\mathrm{MnTe}_{2}$ at the interface. The shift of the Te 3d CLBE is likely to due to a change in surface chemistry ( $\mathrm{MnTe}_{2}$ to $\mathrm{MnTe}$ ) rather than band bending. Using the valence band maximum and the binding energy (BE) of the In $3 \mathrm{~d}$ and $\mathrm{Zn} 2 \mathrm{p}$ core levels at intermediate coverage the valence band offset can be estimated to be on the order of $2 \mathrm{eV}$.
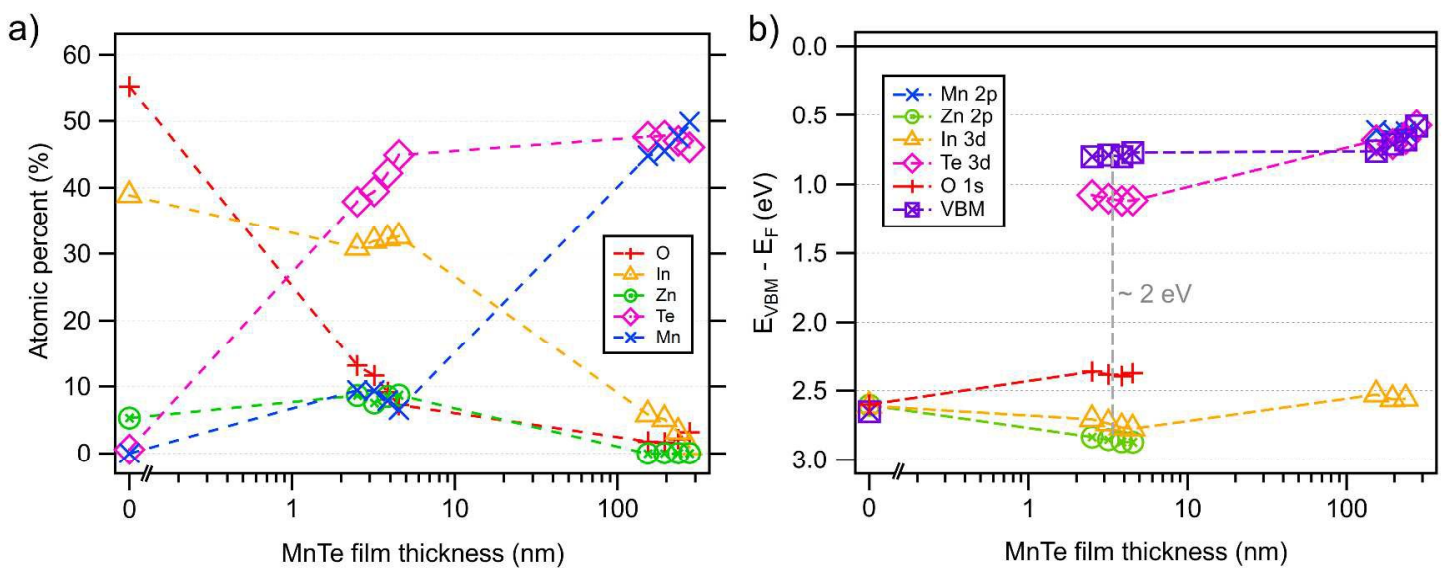

Figure 6: XPS analysis of the Inter-tool a-IZO/MnTe interface experiment. a) Composition as a function of MnTe film thickness. In as well as Zn diffusion can be observed during film growth. 
b) Evolution of the core level binding energies. Non-parallel shifts that correspond to elements within the same material system indicate interface reactions and changes in surface chemistry.

In addition to the XPS measurements at each deposition step, ultraviolet photoelectron spectroscopy (UPS) measurements were carried out on the plain a-IZO substrate and the last deposition step, to determine the work function $(\Phi)$ of both the a-IZO substrate and the MnTe film. Figure 7a shows UPS measurements of the respective materials. By subtracting the BE for the secondary electron edge from the excitation energy (He I: $h v=21.22 \mathrm{eV}$ ) the work functions for a-IZO and MnTe at the free surface were determined to be $\Phi_{\mathrm{a}-\mathrm{IZO}}=3.8 \mathrm{eV}$ and $\Phi_{\mathrm{MnTe}}=4.2$ $\mathrm{eV}$, respectively. With these values and the band gaps from optical spectroscopy (Figure 3) a schematic band diagram can be drawn (see Figure 7c).

a)

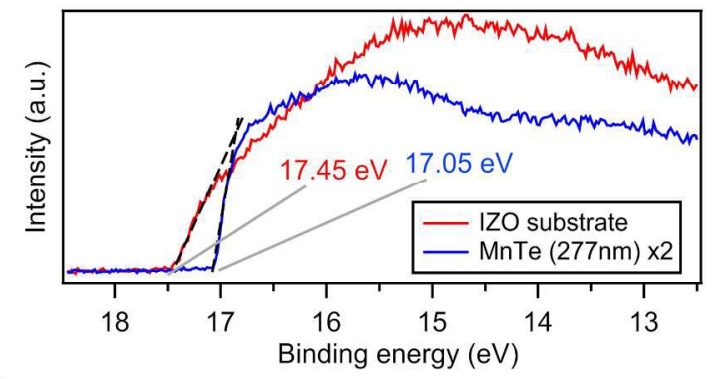

b)

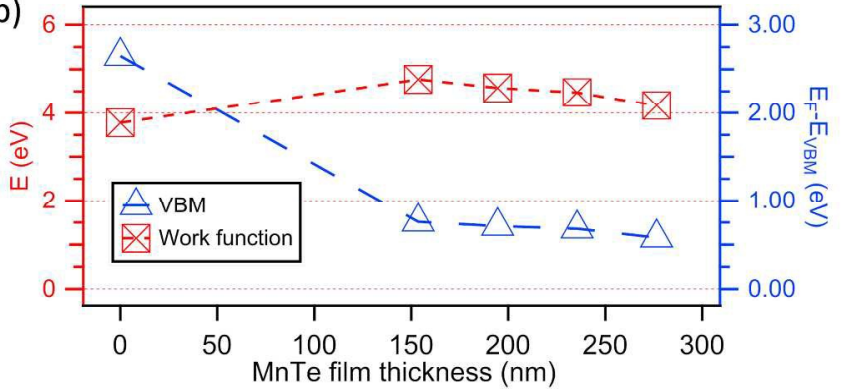

c)

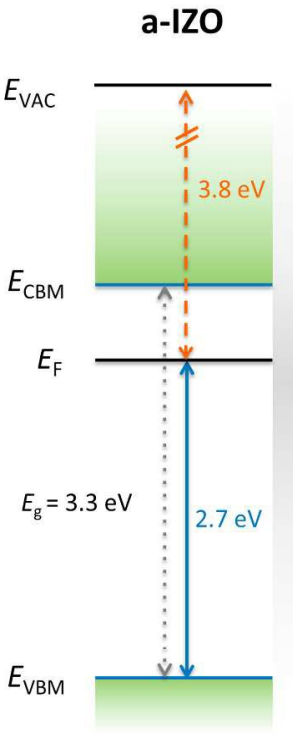

WZ-MnTe

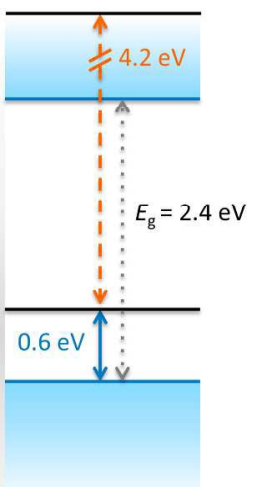

Figure 7: a) UPS analysis of both a-IZO and MnTe surfaces. The work function can be derived from the secondary electron edge b) Work function $\Phi$ from UPS and VBM from XPS as a function of MnTe film thickness c) Schematic of the band alignment at the a-IZO/MnTe interface. Inter-diffusion of $\mathrm{In}$ and $\mathrm{Zn}$ at the interface as well as the associated interface reactions prevent reliable determination of the valence band offset as well as the band bending in either material.

In order to provide ideal band alignment for hole-transport it is beneficial if the ionization potentials of the p-type absorber and the back contact layer line up. The ionization potential $I_{\mathrm{P}}=$ 
$\Phi+E_{\mathrm{VBM}}$ for the WZ-type MnTe layer could be determined to be in the order of $5 \mathrm{eV}$ (see Figure 7b). This is slightly lower than ionization potentials for common p-type thin film photovoltaic absorbers $\left(\mathrm{Cu}_{2} \mathrm{~S}, \mathrm{Cu}_{2} \mathrm{O}: I_{\mathrm{P}} \sim 5.4 \mathrm{eV}^{21-24}\right.$; CdTe: $\left.I_{\mathrm{P}}: \sim 5.7 \mathrm{eV}^{16,25}\right)$. While part of this mismatch could be compensated by band bending in either film, a small valence band offset might remain resulting in a barrier for hole transport and increased recombination. Alloying of MnTe with other II-VI materials may provide a promising route to preserve the WZ-structure of the material while increasing the ionization potential ${ }^{26}$. In addition, such alloying or extrinsic doping may allow control of the doping level to further improving the interface charge transport properties.

\section{Discussion}

In this work the WZ polymorph of MnTe was stabilized using non-equilibrium sputtering on aIZO substrates. Wide-angle XRD measurements unambiguously confirm the WZ structure of the MnTe films (Figure 2a). The film thicknesses well above $600 \mathrm{~nm}$ indicate that the stabilization mechanism is not strain related; neither is it due to charge transfer from the substrate as indicated by $\mathrm{NC}-\mathrm{MnTe}$ deposition on both $\mathrm{Al}$ and $\mathrm{Au}$ with different work functions. Instead, the results of TEM and XPS measurements suggest that inter-diffusion at the interface plays an important role in the stabilization of this WZ polymorph on MnTe. Both EDS and XPS measurements clearly indicate diffusion of In and $\mathrm{Zn}$ into the MnTe film. At the same time EDS measurements on cross-sections of MnTe layers on a-IZO show a thin interfacial layer of Mn. XPS measurements indicate the presence of metallic $\mathrm{Mn}$ at the interface. Together this could be interpreted as diffusion of $\mathrm{Mn}$ into the a-IZO layer leading to Mn-poor compositions over the first few $\mathrm{nm}$ of the MnTe film, being consistent with the presence of $\mathrm{MnTe}_{2}$ as observed in SAED patterns.

There are several possible interdiffusion based mechanisms that could lead to stabilization of WZ-MnTe on a-IZO substrates. First, alloying MnTe with ZnTe which is known to crystallize in $\mathrm{ZB}$ and $\mathrm{WZ}$ structure, ${ }^{26}$ may lead to formation of $\mathrm{WZ}-\mathrm{Mn}_{1-\mathrm{x}} \mathrm{Zn}_{\mathrm{x}} \mathrm{Te}$ alloys at the interface, which in turn can lead to templating of WZ-MnTe on these seed layers. A similar templating mechanism has been described in literature for ZB-MgS polymorph growth on GaAs substrates $^{27}$. Second, the diffusion of $\mathrm{Mn}$ into the a-IZO leading to a Te-rich interface region and 
the formation of PY-type $\mathrm{MnTe}_{2}$, which perhaps could also trigger the growth of WZ-type MnTe. The dominant stabilization mechanism of the WZ phase, however, remains unknown and warrants further investigations that are beyond the scope of this work. If such mechanism could be isolated, it may become possible to grow WZ-MnTe on any substrate, facilitating the use of this material for CdTe back contacts and other applications.

In addition, the possible role of trace amounts of In during the nucleation and growth process has yet to be fully understood. From TEM measurements of MnTe on a-IZO two growth regions can be identified (Figure S1). The crystallinity of the MnTe film seems to increase after the initial 100-200nm of the MnTe film, which corresponds well with the diffusion depth of In (Figure 4h). In this context it is unclear if the presence of In is suppressing the formation of larger crystallites or if smaller amounts of secondary phases are formed. Further research would be needed to understand the In-related mechanisms of WZ-MnTe stabilization on amorphous IZO substrates.

\section{Summary}

In summary we stabilized a previously unreported wurtzite polymorph of the semiconducting compound MnTe by sputter deposition on amorphous indium zinc oxide (a-IZO). The stabilization mechanism appears not to be limited to a critical film thickness, and not influenced by the work function of the substrate. The stabilization is likely related to interdiffusion at the WZ-MnTe/a-IZO interface, but the exact mechanism of the stabilization remains to be pinpointed. The WZ-type MnTe films show properties that are strikingly different from the ground state NC polymorph of MnTe - in particular the increase in optical band gap is remarkable. The combination of this $>2.4 \mathrm{eV}$ optical band gap and p-type doping make this material a potential candidate for future applications as a p-type contact material in optoelectronic energy conversion devices such as solar cells or light emitting diodes.

\section{Methods}

\subsection{Combinatrial thin film deposition}

The deposition of the MnTe combinatorial libraries was carried out through RF magnetron sputtering in a custom UHV sputter tool (AJA International) with a base pressure of $2 \times 10^{-7}$ 
mbar. Various substrates were used including Eagle XG (EXG) borosilicate glass (Corning) and Indium Zinc Oxide (a-IZO) coated EXG glass (more information on the a-IZO deposition can be found in the literature ${ }^{19}$ ). The substrates were heated through a partial thermal contact with a heated sample holder. This setup resulted in a temperature gradient across the library ranging from $550{ }^{\circ} \mathrm{C}$ on the hottest, to $320{ }^{\circ} \mathrm{C}$ on the coldest side. This setup resulted in a temperature gradient across the substrate covering multiple deposition temperatures in a single experiment.

The a-IZO used in this study has been shown to exert remarkable thermal stability ${ }^{19}$. However substrate temperatures in excess of $550^{\circ} \mathrm{C}$ led to phase impurities (see also Figure S3) and appearance of the nanocrystallites of the amorphous under-layer. For this reason more moderate deposition temperatures of $320^{\circ} \mathrm{C}$ were chosen for this study.

An orthogonal deposition rate gradient was achieved with a deposition angle of roughly $30^{\circ}$ and a target to substrate distance of $10 \mathrm{~cm}$. The sputter gun was equipped with a 2 " ceramic MnTe target (99.9\% purity, Advanced Engineering Materials Ltd), respectively. Pure Argon (99.99\% purity) was used as process gas. The process pressure was kept at $2.0 \times 10^{-3}$ mbar for the duration of the deposition, as well as for the subsequent cooling step. Depending on the position on the substrate, deposition rates between $2.5 \mathrm{~nm} / \mathrm{min}$ and $4.5 \mathrm{~nm} / \mathrm{min}$ were achieved. The deposition times were chosen to produce film thicknesses in the range of $500 \mathrm{~nm}$ to $700 \mathrm{~nm}$. More details about deposition procedures in the same chamber can be found elsewhere ${ }^{28}$.

\subsection{Spatially-resolved characterization}

The MnTe libraries were investigated in regard to structural, compositional and optoelectronic properties using spatially resolved X-ray diffraction (XRD), X-ray fluorescence (XRF), UV-Vis measurements. The XRD measurements were performed with a Bruker D8 Discover x-ray diffractometer with a Vantec 500 area detector in approximately a two-theta geometry using $\mathrm{Cu} \mathrm{K \alpha}$ radiation. The composition and thickness were mapped using x-ray fluorescence (XRF, Fischerscope XDV-SDD). UV-Vis transmission and reflection measurements were taken in a custom setup covering a wavelength range from $300 \mathrm{~nm}$ to $1100 \mathrm{~nm}$. All combinatorial data sets were evaluated with Igor Pro software using customized routines. A more detailed description of the characterization procedures can be found in previous works. $^{29}$ The deposition and characterization data will be made available at https://htem.nrel.gov ${ }^{30}$ 


\subsection{Electron microscopy analysis}

Transmission electron microscopy (TEM) micrographs were acquired with an FEI Co. Talos F200X transmission electron microscope with scanning capabilities operating at an accelerating voltage of $200 \mathrm{keV}$. Specimens for TEM were prepared from deposited films via in-situ focused ion beam lift-out methods using an FEI Co. Helios Nanolab 600i SEM/FIB DualBeam workstation $^{31}$. Specimens were ion milled at $2 \mathrm{keV}$ and $77 \mathrm{pA}$ to remove Ga ion beam damage and achieve a final thickness of approximately $80 \mathrm{~nm}$. Structural characterization was conducted by acquiring selected area electron diffraction (SAED) patterns on an FEI Co. Ceta 16M pixel CMOS camera at a camera length of $410 \mathrm{~mm}$. Platinum from the FIB was used to calibrate the camera constant, allowing SAED reflections to be accurately measured and indexed. Chemical mapping was performed in the TEM using the Super-X energy-dispersive X-ray spectroscopy (EDS) system equipped with four windowless silicon drift detectors, allowing for high resolution and count rates. More details on TEM/EDS measurements have been previously published ${ }^{32}$.

\subsection{Photoelectron spectroscopy characterization}

Photoemission spectra were acquired on a Kratos AXIS Nova spectrometer calibrated to the core level positions of sputter-cleaned metal $(\mathrm{Au}, \mathrm{Ag}, \mathrm{Cu}, \mathrm{Mo})$ surfaces. Samples were transferred between the sputter growth chamber and the XPS instrument in a custom-built highvacuum transport pod within NREL's Process Development and Integration Laboratory (PDIL). XP spectra were taken using monochromated Al $\mathrm{K} \alpha$ radiation $(1486.7 \mathrm{eV})$ for excitation at a nominal resolution of $400 \mathrm{meV}$ (pass energy $10 \mathrm{eV}$, step size $0.025 \mathrm{eV}$, dwell time $120 \mathrm{~ms}$, spot size $\left.700 \mu \mathrm{m}^{2}\right)$. UP spectra were acquired from the He I (21.22 eV) excitation line at an experimental resolution below $150 \mathrm{meV}$. The acquired spectra were all referenced to the previously determined Fermi level of the sputter-cleaned metal calibration samples. Four measurement spots corresponding to different film thicknesses were defined on the combinatorial library. A more detailed description of the Combinatorial PES interface experiment can be found in our previous works ${ }^{33}$.

\section{Acknowledgements}


This work was supported by the U.S. Department of Energy under Contract No. DE-AC3608GO28308 with Alliance for Sustainable Energy, LLC, the Manager and Operator of the National Renewable Energy Laboratory. Funding provided by Office of Science, Office of Basic Energy Sciences, as part of the Energy Frontier Research Center "Center for Next Generation of Materials Design: Incorporating Metastability". Yanbing Han is supported from Science and Technology Commission of Shanghai Municipality (No. 16JC1400603), a grant from the National Natural Science Foundation of China (No. 61471126), and a stipend from China Scholarship Council. Multiple useful discussions with Stephan Lany are gratefully acknowledged. The authors declare no competing financial interests. The views expressed in the article do not necessarily represent the views of the DOE or the U.S. Government. The U.S. Government retains and the publisher, by accepting the article for publication, acknowledges that the U.S. Government retains a nonexclusive, paid-up, irrevocable, worldwide license to publish or reproduce the published form of this work, or allow others to do so, for U.S. Government purposes.

\section{References}

(1) Madelung, O. Semiconductor: Data Handbook, 3rd ed.; Springer-Verlag: Berlin, 2004.

(2) Wang, J.; Isshiki, M. Wide-Bandgap II--VI Semiconductors: Growth and Properties. In Springer Handbook of Electronic and Photonic Materials; Kasap, S., Capper, P., Eds.; Springer US: Boston, MA, 2007; pp 325-342.

(3) Paszkowicz, W.; Dynowska, E. High Pressure - High Temperature Diffraction Study of MnTe Using Synchrotron Radation. Acta Physica Polonica A 1997, 91 (5), 939-944.

(4) Mimasaka, M.; Sakamoto, I.; Murata, K.; Fujii, Y.; Onodera, A. Pressure-Induced Phase Transitions of MnTe. J. Phys. C: Solid State Phys. 1987, 20, 4689-4694.

(5) Iwanowski, R. J.; Heinonen, M. H.; Janik, E. X-Ray Photoelectron Spectra of Zinc-Blende MnTe. Chemical Physics Letters 2004, 387 (1-3), 110-115.

(6) Furdyna, J. K. Diluted Magnetic Semiconductors. Journal of Applied Physics 1988, 64 (4), 29-64. 
(7) Dietl, T. A Ten-Year Perspective on Dilute Magnetic Semiconductors and Oxides. Nature Materials 2010, 9 (12), 965-974.

(8) Buller, B.; Gloeckler, M.; Gupta, A.; Powell, R.; Shao, R.; Xiong, G.; Yu, M. L.; Zhao, Z. Photovoltaic Device and Methods of Forming the Same; US20170288073 A1; First Solar, Inc.: USA, 2017.

(9) Major, J. D.; Phillips, L. J.; Al Turkestani, M.; Bowen, L.; Whittles, T. J.; Dhanak, V. R.; Durose, K. P3HT as a Pinhole Blocking Back Contact for CdTe Thin Film Solar Cells. Solar Energy Materials and Solar Cells 2017, 172, 1-10.

(10) Kaznacheyev, K. V.; Muro, T.; Matsushita, T.; Iwasaki, T.; Kuwata, Y.; Harada, H.; Suga, S.; Ishii, H.; Miyahara, T.; Mizokawa, T.; et al. Electronic Structure of Pyrite-Type MnTe 2 Studied by Photoelectron Spectroscopy. Physical Review B 1998, 58 (20), 1349113497.

(11) Krause, M.; Bechstedt, F. Structural and Magnetic Properties of MnTe Phases from Ab Initio Calculations. Journal of Superconductivity and Novel Magnetism 2013, 26, 19631972.

(12) NREL Materials Database http://materials.nrel.gov/ (accessed Apr 5, 2018).

(13) Siol, S.; Holder, A.; Ortiz, B. R.; Parilla, P. A.; Toberer, E.; Lany, S.; Zakutayev, A. Solubility Limits in Quaternary SnTe-Based Alloys. RSC Advances 2017, 7 (40), 2474724753.

(14) Siol, S.; Holder, A.; Steffes, J.; Schelhas, L. T.; Stone, K. H.; Garten, L.; Perkins, J. D.; Parilla, P. A.; Toney, M. F.; Huey, B. D.; et al. Negative-Pressure Polymorphs Made by Heterostructural Alloying. Science Advances 2018, 4 (4), eaaq1442.

(15) Hoye, R. L. Z.; Schulz, P.; Schelhas, L. T.; Holder, A. M.; Stone, K. H.; Perkins, J. D.; Vigil-Fowler, D.; Siol, S.; Scanlon, D. O.; Zakutayev, A.; et al. Perovskite-Inspired Photovoltaic Materials: Toward Best Practices in Materials Characterization and Calculations. Chemistry of Materials 2017, 29 (5), 1964-1988.

(16) Ellmer, K.; Klein, A.; Rech, B. Transparent Conductive Zinc Oxide; Springer-Verlag: Heidelberg, 2008. 
(17) Fioretti, A. N.; Zakutayev, A.; Moutinho, H.; Melamed, C.; Perkins, J. D.; Norman, A. G.; Al-Jassim, M.; Toberer, E. S.; Tamboli, A. C. Combinatorial Insights into Doping Control and Transport Properties of Zinc Tin Nitride. Journal of Materials Chemistry C 2015, 3, $11017-11028$.

(18) Xie, W.; Populoh, S.; Gałązka, K.; Xiao, X.; Sagarna, L.; Liu, Y.; Trottmann, M.; He, J.; Weidenkaff, A. Thermoelectric Study of Crossroads Material MnTe via Sulfur Doping. Journal of Applied Physics 2014, 115 (10), 103707.

(19) Taylor, M. P.; Readey, D. W.; van Hest, M. F. A. M.; Teplin, C. W.; Alleman, J. L.; Dabney, M. S.; Gedvilas, L. M.; Keyes, B. M.; To, B.; Perkins, J. D.; et al. The Remarkable Thermal Stability of Amorphous In-Zn-O Transparent Conductors. Advanced Functional Materials 2008, 18 (20), 3169-3178.

(20) Nesbitt, H. W.; Banerjee, D. Interpretation of XPS Mn(2p) Spectra of Mn Oxyhydroxides and Constraints on the Mechanism of MnO2 Precipitation. American Mineralogist 1998, $83(3-4), 305-315$.

(21) Siol, S. Kupfer-Chalkogenide Für Photovoltaische Anwendungen, Technische Universität: Darmstadt, 2014.

(22) Deuermeier, J.; Gassmann, J.; Brötz, J.; Klein, A. Reactive Magnetron Sputtering of $\mathrm{Cu} 2 \mathrm{O}$ : Dependence on Oxygen Pressure and Interface Formation with Indium Tin Oxide. Journal of Applied Physics 2011, 109 (11), 113704.

(23) Türck, J.; Siol, S.; Mayer, T.; Klein, A.; Jaegermann, W. Cu2S as Ohmic Back Contact for CdTe Solar Cells. Thin Solid Films 2015, 582, 336-339.

(24) Türck, J.; Nonnenmacher, H.-J.; Connor, P. M. L.; Siol, S.; Siepchen, B.; Heimfarth, J. P. J. P.; Klein, A.; Jaegermann, W. Copper (I) Oxide (Cu2O) Based Back Contact for P-I-N CdTe Solar Cells. Progress in Photovoltaics: Research and Applications 2016, 15 (9), 659-676.

(25) Teeter, G. X-Ray and Ultraviolet Photoelectron Spectroscopy Measurements of Cu-Doped CdTe(111)-B: Observation of Temperature-Reversible CuxTe Precipitation and Effect on Ionization Potential. Journal of Applied Physics 2007, 102 (3), 34504. 
(26) Szwacki, N. G.; Przeździecka, E.; Dynowska, E.; Bogusławski, P.; Kossut, J. Structural Properties of MnTe, ZnTe, and ZnMnTe. Acta Physica Polonica A 2004, 106 (2), 233238.

(27) Rajan, A.; Moug, R. T.; Prior, K. A. Growth and Stability of Zinc Blende MgS on GaAs, GaP, and InP Substrates. Applied Physics Letters 2013, 102 (3).

(28) Han, Y.; Siol, S.; Zhang, Q.; Zakutayev, A. Optoelectronic Properties of Strontium and Barium Copper Sulfides Prepared by Combinatorial Sputtering. Chemistry of Materials 2017, 29 (19), 8239-8248.

(29) Siol, S.; Dhakal, T. P.; Gudavalli, G. S.; Rajbhandari, P. P.; DeHart, C.; Baranowski, L. L.; Zakutayev, A. Combinatorial Reactive Sputtering of In2S3 as an Alternative Contact Layer for Thin Film Solar Cells. ACS Applied Materials \& Interfaces 2016, 8 (22), 14004-14011.

(30) Zakutayev, A.; Wunder, N.; Schwarting, M.; Perkins, J. D.; White, R.; Munch, K.; Tumas, W.; Phillips, C. An Open Experimental Database for Exploring Inorganic Materials. Scientific Data 2018, 5, 180053.

(31) Giannuzzi, L. A.; Stevie, F. A. A Review of Focused Ion Beam Milling Techniques for TEM Specimen Preparation. Micron 1999, 30 (3), 197-204.

(32) Bikowski, A.; Siol, S.; Gu, J.; Holder, A.; Mangum, J. S. J. S.; Gorman, B.; Tumas, W.; Lany, S.; Zakutayev, A. Design of Metastable Tin Titanium Nitride Semiconductor Alloys. Chemistry of Materials 2017, 29 (15), 6511-6517.

(33) Siol, S.; Schulz, P.; Young, M.; Borup, K. A.; Teeter, G.; Zakutayev, A. Combinatorial In Situ Photoelectron Spectroscopy Investigation of Sb2Se3/ZnS Heterointerfaces. Advanced Materials Interfaces 2016, 3 (24), 1600755. 


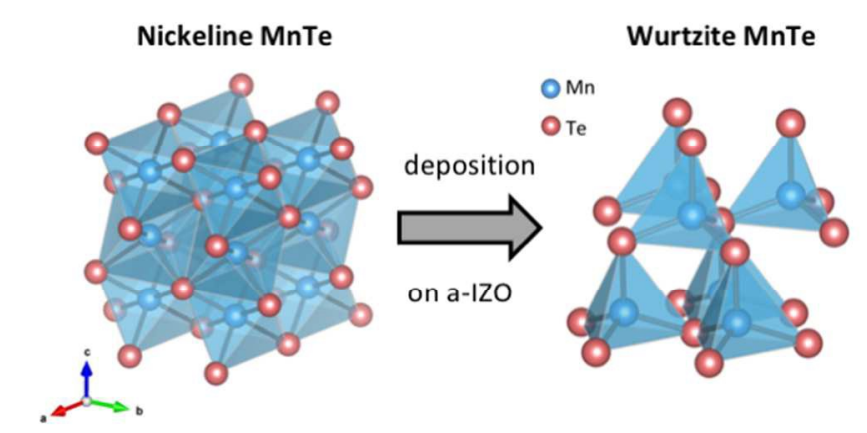

Wurtzite polymorph of MnTe with wider band gap and moderate p-type doping is stabilized on amorphous indium zinc oxide substrate 